\title{
O papel de processos relacionados com a regulação emocional e da vergonha associada à doença crónica nos sintomas de depressão, ansiedade e stress de pessoas com doença celíaca
}

\section{The role of emotion regulation related processes and chronic illness-related shame in depression, anxiety and stress symptoms of people facing coeliac} disease

\author{
Melanie Fernandes (1) \\ Ana Galhardo (1, 2) \\ Ilda Massano-Cardoso (1,3,4) \\ (1) Inst Sup Miguel Torga, Coimbra, Portugal \\ (2) Univ Coimbra, CINEICC, FPCEUC, Portugal \\ (3) Univ Coimbra, FMUC, Portugal \\ (4) Univ Coimbra, CEISUC, FEUC, Portugal
}

Recebido: 13/12/2019; Revisto: 06/03/2020; Aceite: 08/04/2020.

https://doi.org/10.31211/rpics.2020.6.1.162

\section{Resumo}

Objetivo: O presente estudo, de carácter exploratório, teve como principal objetivo examinar o papel preditor de processos relacionados com a regulação emocional (fusão cognitiva, evitamento experiencial, autocompaixão e autojulgamento) e da vergonha associada à doença nos sintomas psicopatológicos de depressão, ansiedade e stress em pacientes com diagnóstico de doença celíaca. Métodos: Através de uma associação de pacientes, foram recrutados 67 sujeitos com diagnóstico de doença celíaca autorreportado, os quais completaram online um questionário sociodemográfico e clínico e um conjunto de instrumentos de autorresposta, mais precisamente as Escalas de Ansiedade, Depressão e Stress - 21 (EADS-21), o Cognitive Fusion Questionnaire - Chronic IIIness (CFQ-CI), o Acceptance and Action Questionnaire-II (AAQ-II), a Self-Compassion Scale (SCS), e a Chronic Illness-related Shame Scale (CISS). O papel mediador dos processos relacionados com a regulação emocional e da vergonha associada à doença crónica foi analisado através do cálculo de regressões lineares múltiplas hierárquicas. Resultados: O índice compósito de autojulgamento (autocriticismo, isolamento e sobreidentificação) revelou-se como o único preditor significativo dos sintomas de depressão, ansiedade e stress em pessoas com doença celíaca. Conclusões: Nas intervenções psicológicas dirigidas a pacientes com doença celíaca a avaliação e integração do autojulgamento enquanto processo de regulação emocional poderá ser relevante para a obtenção de ganhos terapêuticos no que se refere aos sintomas emocionais negativos de depressão, ansiedade e stress.

Palavras-Chave: Doença celíaca; Regulação emocional; Sintomas psicopatológicos; Vergonha associada à doença crónica.

\section{DI\&D | ISMT \\ rpics@ismt.pt \\ https://rpics.ismt.pt}

Publicação em Acesso Aberto

(C)2020. O(s) Autor(es). Este é um artigo de acesso aberto distribuído sob a Licença Creative Commons Attribution, que permite uso, distribuição e reprodução sem restrições em qualquer meio, desde que o trabalho original seja devidamente citado.
Ana Galhardo

ISMT

Instituto Superior Miguel Torga

Largo da Cruz de Celas, n¹, 3000-132

Coimbra, Portugal.

Email: anamargaridagalhardo@gmail.com 


\begin{abstract}
Aim: The current exploratory study sought out to examine the predictive role of emotion regulation related processes (cognitive fusion, experiential avoidance, self-compassion, and self-judgment) and chronic illness-related to shame in the psychopathological symptoms of depression, anxiety and stress in patients presenting a diagnosis of coeliac disease. Method: Sixty-seven participants with self-reported coeliac disease were recruited through a patients' association and completed an online sociodemographic and clinical questionnaire and a set of self-report instruments, namely the Anxiety, Depression, and Stress Scales - 21 (DASS-21), the Cognitive Fusion Questionnaire - Chronic Illness (CFQ-CI), the Acceptance and Action Questionnaire-II (AAQ-II), the Self-Compassion Scale (SCS), and the Chronic Illness-related Shame Scale (CISS). The mediating role of the emotion regulation related processes and the chronic illness-related shame was analyzed by calculating hierarchical multiple linear regressions. Results: The self-judgment composite index (self-criticism, isolation, and overidentification) was the only significant predictor of depression, anxiety, and stress symptoms in people with coeliac disease. Conclusions: In psychological interventions designed for patients with coeliac disease, assessment and clinical work targeting self-judgment as an emotion regulation process may be relevant to promote therapeutic gains regarding the negative emotional symptoms of depression, anxiety, and stress.
\end{abstract}

Keywords: Coeliac disease; Chronic illness-related shame; Emotion regulation; Psychopathological symptoms.

\title{
Introdução
}

\section{Doença Celíaca}

A Doença Celíaca (DC) é tida como uma doença crónica, autoimune, que se caracteriza por alterações na absorção dos nutrientes decorrentes da destruição das vilosidades intestinais quando é ingerido glúten. A DC tem uma componente hereditária, pelo que os sujeitos com antecedentes familiares têm uma maior probabilidade de vir a sofrer de DC (Butterworth \& Los, 2019).

De acordo com a Associação Portuguesa de Celíacos (APC), na Europa a DC está presente em 1\% da população (Associação Portuguesa de Celíacos [APC], 2019). Por sua vez, os dados de prevalência em Portugal indicam que entre 1\% a 3\% da população apresenta DC. No entanto, estima-se que existam entre 70.000 a 100.000 pacientes com DC sem diagnóstico em Portugal (APC, 2019). De acrescentar que existe uma maior prevalência de DC no sexo feminino (Mariné et al., 2011). No entanto, Bai et al. (2005) referem que no sexo masculino o diagnóstico efetivo tende a ser estabelecido mais rapidamente, possivelmente pelo facto de os homens terem propensão para apresentar um maior número de sintomas adversos da absorção deficitária.

Melicharova et al. (2016) sugerem que, na etapa de diagnóstico, a aceitação, em muitos casos, não é imediata e os pacientes manifestam dúvidas em relação à doença, podendo também evidenciar sentimentos negativos decorrentes do carácter invasivo das técnicas de diagnóstico utilizadas. De acrescentar que a DC sem diagnóstico, ou sem tratamento, pode acarretar uma multiplicidade de outras condições clínicas (e.g., anemia ferropénica; osteoporose de início precoce ou osteopenia; infertilidade e aborto espontâneo; intolerância à lactose; deficiências de vitaminas e minerais; distúrbios do sistema nervoso central e periférico; insuficiência pancreática) (Celiac Disease Foundation [CDF], 2019). Além disso, é também de referir a existência de várias doenças comórbidas, como anemia, doença autoimune da tiroide, dermatite herpetiforme, ataxia de glúten, cardiomiopatia dilatada idiopática, síndrome de fadiga crónica, hepatite autoimune, doença hepática, colite linfocítica, colite microscópica, neuropatia periférica, cirrose biliar primária, síndrome de Sjögren, diabetes tipo I e infertilidade inexplicada (CDF, 2019). 
O tratamento da DC consiste na realização de uma dieta isenta de glúten (DIG), a qual visa evitar outras consequências no decorrer da doença (Butterworth \& Los, 2019; Leonard et al., 2017) e aumentar a qualidade de vida (Nachman et al., 2010). Na maioria dos pacientes são observadas melhorias após duas a três semanas do início de uma DIG (Catassi \& Lionetti, 2020). De acordo com o National Canadian Survey (Zarkadas et al., 2006), o ano que se segue ao diagnóstico tende a ser aquele em que se verifica uma pior qualidade de vida, em virtude da introdução de uma DIG e das mudanças no estilo de vida que esta exige. Hall et al. (2009) sublinham precisamente a existência de dificuldades no cumprimento de uma DIG por parte dos pacientes. Dowd et al. (2015) indicam que a eficácia autorreguladora se constitui como um construto relevante no que se refere ao cumprimento da DIG em pacientes com DC.

\section{Aspetos psicológicos na doença celíaca}

A DC pode igualmente traduzir-se em consequências significativas do ponto de vista do bem-estar psicológico. Estas poderão, por um lado, estar relacionadas com a doença e o seu impacto bioquímico e, por outro, com a vivência do paciente, não apenas da própria doença, mas também do respetivo tratamento (Zingone et al., 2015). Com efeito, Siniscalchi et al. (2005) reportaram a existência de relação entre depressão e sintomas de fadiga em pacientes com DC que ainda não haviam iniciado tratamento. De um modo geral, os sujeitos com DC tendem a apresentar maiores níveis de ansiedade, depressão e stress comparativamente com adultos saudáveis (Zingone et al., 2015). No entanto, esses sintomas podem sofrer flutuações em função das diferentes fases do diagnóstico. Previamente ao conhecimento do diagnóstico de DC, são frequentes sentimentos de preocupação sobre os sintomas (que não são compreendidos), e desapontamento com consultas médicas (sem obtenção de informação concreta). Uma vez definido o diagnóstico, as preocupações tendem a centrar-se em conteúdos relativos ao modo como vão lidar com a doença a longo prazo, experienciando simultaneamente sentimentos de alívio pelo facto de, finalmente, saberem qual a sua doença (Zingone et al., 2015). Em alguns pacientes as dificuldades psicológicas atenuam-se à medida que aumenta o seu conhecimento acerca da doença, mas noutros as dificuldades de adaptação e adesão à DIG, assim como a adaptação em termos sociais, persistem (Ludvigsson et al., 2015; Zingone et al., 2015).

Efetivamente, os sintomas de ansiedade e depressão podem exercer influência na adesão a uma DIG e na qualidade de vida (Zingone et al., 2015). Segundo Smith e Gerdes (2012), a prevalência e severidade da depressão é superior em pacientes com DC quando comparados com grupos de controlo sem a doença. Nos pacientes com DC a depressão pode advir dos sintomas físicos e/ou das restrições/impedimentos sociais que a doença crónica implica (Smith \& Gerdes, 2012). Cossu et al. (2017) referem que os sintomas depressivos podem resultar do impacto da doença crónica no bem-estar dos indivíduos, não existindo evidência de uma relação direta entre a depressão e a DC. Por sua vez, a persistência dos sintomas depressivos pode estar associada com uma qualidade de vida mais pobre, e esta, por seu turno, pode resultar das restrições alimentares e do impacto da DC na vida social (Addolorato, 2001). Neste contexto, Zingone e colaboradores (2015) sublinham a importância de os profissionais de saúde atenderem às consequências que a doença encerra, do ponto de vista psicológico, com o intuito de facultar apoio aos pacientes. Assim, é desejável que os pacientes com DC usufruam de acompanhamento psicológico de modo a promover a sua adaptação à DIG, assegurando uma maior adesão a este tratamento e diminuindo a probabilidade de desenvolverem complicações comórbidas (Melicharova et al., 2016; Zarkadas et al., 2006). Esta recomendação é corroborada por Addolorato et al. (2004) que verificaram a existência de uma redução 
dos sintomas depressivos, bem como uma maior adesão à DIG, em pacientes com DC que tiveram apoio psicológico para redução do stress e identificação e esclarecimento de preocupações relativas à DIG, comparativamente com pacientes que integraram um grupo de controlo (treatment as usual).

\section{Processos de regulação emocional e vergonha associada a doença crónica}

Os processos relacionados com a regulação emocional têm vindo a ser apontados como desempenhando um importante papel no desenvolvimento e manutenção de sintomas psicopatológicos (e.g., Hayes et al., 2006; Levin et al., 2014; Masuda et al., 2014). Neste âmbito, e partindo de um enquadramento das terapias cognitivocomportamentais contextuais, construtos como os de evitamento experiencial, fusão cognitiva, autocompaixão e autojulgamento têm suscitado o interesse de investigadores e clínicos, sendo sugeridos como elementos transdiagnósticos, pelo que a sua investigação em pessoas com DC pode contribuir para a inclusão mais fundamentada destes em intervenções psicológicas que lhes sejam dirigidas.

Ao considerar a fusão cognitiva, é possível reconhecer um sobreenvolvimento com o conteúdo dos acontecimentos privados, o que pode conduzir a inflexibilidade psicológica (Gillanders et al., 2014). Nestas circunstâncias, quando o sujeito está excessivamente fusionado, é mais provável que as suas direções de vida valorizadas estejam focadas no controlo ou evitamento de experiências internas desagradáveis (Hayes et al., 2006). No caso da doença crónica, tal pode originar o evitamento de consultas médicas ou a toma da medicação (Trindade, 2018). Com efeito, a fusão cognitiva associada à doença crónica conduz a uma diminuição do bem-estar geral, físico e psicológico (Trindade, Ferreira, \& Pinto-Gouveia, 2018). Dahl et al. (2009) referem que nas situações em que as experiências internas são identificadas como negativas ou adversas, os sujeitos tendem a fusionar-se com essas experiências e a evitar experienciá-las.

Assim, a ligação estreita entre a fusão cognitiva e o evitamento experiencial tem sido amplamente sugerida (Gillanders et al., 2014). O evitamento experiencial é definido como um mecanismo através do qual os sujeitos procuram controlar, alterar ou escapar a experiências internas adversas ou dolorosas (e.g., pensamentos, sensações corporais, emoções, recordações), ainda que estas o possam afastar dos seus valores e ter consequências negativas (Hayes et al., 1996). Ainda que o evitamento experiencial possa, no imediato, ter um efeito positivo, quando utilizado de forma rígida e repetida tende a ter efeitos negativos, afastando o sujeito das ações consistentes com os seus valores (Hayes et al., 2006). De facto, o evitamento experiencial tem vindo a ser indicado como interferindo na qualidade de vida, ansiedade, depressão e autogestão da doença (Chawla \& Ostafin, 2007).

Recentemente a autocompaixão tem sido identificada como um processo fundamental na melhoria do bemestar e na redução do stress em pessoas com um diagnóstico de doença crónica (Sirois \& Rowse, 2016). A autocompaixão engloba uma atitude de compreensão, calor e bondade para consigo mesmo, a capacidade de estar no momento presente, e o reconhecimento da humanidade comum da própria experiência (Neff, 2003). Efetivamente, no âmbito da doença crónica, por exemplo na doença inflamatória do intestino e na artrite, verifica-se que a autocompaixão se associa a estratégias de coping mais adaptativas (Sirois et al., 2015).

Por sua vez, o autojulgamento pode ser concetualizado como a combinação de autocriticismo, sobreidentificação e isolamento (Neff, 2003, 2019). Como tal, quando os sujeitos apresentam níveis elevados de autojulgamento evidenciam uma atitude crítica dirigida a si mesmos perante erros ou inadequações, um sobreenvolvimento com o seu problema e maior dificuldade em perceber a sua experiência como integrando a experiência humana mais 
vasta. Ao considerar especificamente a doença crónica, o autojulgamento relacionado com a condição de doente, as adversidades resultantes da própria doença e das exigências do seu tratamento, e a dificuldade em corresponder às suas expectativas e às das outras pessoas, tendem a aumentar a probabilidade de surgirem sentimentos de vergonha e sintomas psicopatológicos (Trindade, 2018).

Adicionalmente aos processos relacionados com a regulação emocional, um outro construto que tem merecido o interesse dos investigadores é o da vergonha experienciada por pessoas com doença crónica. De acordo com o modelo evolucionário biopsicossocial proposto por Gilbert (2007), a vergonha pode integrar uma dimensão de vergonha externa e uma dimensão de vergonha interna, relacionadas entre si. A vergonha externa é mais focada nos outros, considerando que estes têm uma ideia ou opinião negativa acerca do self (o indivíduo crê existir de uma forma negativa na mente dos outros), tendo a função de regulação das ameaças sociais (Gilbert, 2002). Por sua vez, a vergonha interna é autofocada, e o sujeito apresenta uma visão do self como inferior, defeituoso e/ou sem valor (Gilbert, 2002). Apesar da ligação entre vergonha e psicopatologia (Matos \& Pinto-Gouveia, 2010), a associação entre a vergonha relacionada com a doença crónica e saúde mental poderá ser mediada por processos de regulação das emoções mais específicos. Com efeito, Trindade, Marta-Simões, et al. (2018) referem que a associação entre a vergonha e a saúde psicológica é mediada pela fusão cognitiva associada à doença crónica. Tal significa que quando os pacientes se fusionam com pensamentos cujo conteúdo tem a ver com uma doença crónica, a vergonha tende a exercer um maior efeito na sua saúde mental (Trindade, Marta-Simões et al., 2018). Para além disso, é também importante mencionar que o facto de o paciente evidenciar sintomas físicos pode conduzir a um acréscimo da vergonha relacionada com esses mesmos sintomas e originar autojulgamento (Gilbert, 2007).

Nos últimos anos tem-se verificado um maior interesse pelos aspetos de natureza psicológica no contexto da doença crónica. No entanto, há ainda muitos mecanismos que não se encontram clarificados e que poderão ser úteis, não apenas para uma melhor compreensão das consequências psicológicas destas condições clínicas, mas também para o desenho de intervenções mais específicas, com vista a diminuir o potencial impacto negativo, em termos psicológicos, da doença crónica. Assim, o presente estudo procurou examinar o papel de processos relacionados com a regulação emocional (fusão cognitiva, evitamento experiencial, autocompaixão e autojulgamento) e da vergonha relacionada com a doença crónica enquanto preditores dos sintomas psicopatológicos de depressão, ansiedade e stress em pessoas com doença celíaca.

\section{Método}

\section{Participantes}

O presente estudo foi desenvolvido em pessoas com idades compreendidas entre os 18 e os 69 anos, de ambos os sexos. A amostra foi constituída por 67 sujeitos, sendo que 60 (89,6\%) eram do sexo feminino e sete (10,4\%) do sexo masculino, todos eles com o diagnóstico de doença celíaca autorreportado (não existiu consulta de processos médicos). Nos 67 participantes, a média de idades foi de 36,99 anos $(D P=11,23)$. Trinta e oito (56,7\%) sujeitos eram casados(as)/viviam em união de facto, 26 (38,8\%) eram solteiros, dois (3,0\%) eram divorciados(as) e apenas um indivíduo (1,5\%) era viúvo. Os participantes habitavam maioritariamente em meio urbano ( $n=54$; $80,6 \%)$ e $13(19,4 \%)$ residiam em meio rural. Relativamente aos anos de escolaridade, observou-se uma média de $14,96(D P=3,17)$. Quanto à situação laboral, 50 participantes $(74,6 \%)$ encontravam-se empregados, oito 
$(11,9 \%)$ eram estudantes, seis (9,0\%) estavam desempregados e, por último, três (4,5\%) encontravam-se já reformados. Os participantes haviam sido diagnosticados com doença celíaca há no mínimo um e no máximo 37 anos, correspondendo a uma média de 9,04 ( $D P=9,90)$ anos. Este diagnóstico foi realizado, em média, quando tinham 28,19 $(D P=15,57)$ anos de idade. Apenas três participantes $(4,5 \%)$ referiram ter recorrido a acompanhamento psicológico relacionado com a doença.

\section{Instrumentos}

De modo a obter informação acerca de variáveis sociodemográficas e clínicas foi elaborado especificamente para este estudo um questionário sociodemográfico e clínico. Em relação aos restantes instrumentos de autorresposta que foram integrados no protocolo de avaliação, realizou-se a seleção com base nos objetivos do estudo.

Questionário sociodemográfico e clínico. O questionário sociodemográfico englobou as seguintes variáveis: sexo, idade, estado civil, zona de residência, anos de escolaridade concluídos e situação laboral. De forma a obter informações clínicas acerca da doença celíaca foi definido um conjunto de questões: "Há quanto tempo foi diagnosticado/a com doença celíaca?"; “Que idade tinha quando a doença foi diagnosticada” e, por fim, "Tem ou teve acompanhamento psicológico relacionado com a Doença Celíaca?”.

Escalas de Ansiedade, Depressão e Stress - 21 (EADS-21; Depression, Anxiety and Stress Scale, Lovibond \& Lovibond, 1995; versão Portuguesa de Pais-Ribeiro et al., 2004). A EADS-21 é composta por 21 itens distribuídos por três subescalas, nomeadamente de depressão (e.g., "Não consegui sentir nenhum sentimento positivo"), ansiedade (e.g., "Senti dificuldades em respirar") e stress (e.g., "Tive tendência a reagir em demasia em determinadas situações"). É solicitado aos respondentes que indiquem o grau em que cada uma das afirmações se Ihes aplicou "na semana passada", utilizando uma escala de resposta de quatro opções: 1. "Não se aplicou nada a mim", 2. "Aplicou-se a mim algumas vezes", 3. "Aplicou-se a mim muitas vezes", 4. "Aplicou-se a maior parte das vezes". Pontuações mais altas em cada subescala sugerem estados afetivos mais negativos. Na versão portuguesa os valores da consistência interna foram de 0,85 para a subescala de depressão, de 0,74 para a subescala de ansiedade, e 0,81 para a subescala de stress (Pais-Ribeiro et al., 2004). No presente estudo os valores de alfa de Cronbach encontrados foram de 0,93 (depressão), 0,78 (ansiedade) e 0,92 (stress).

Cognitive Fusion Questionnaire - Chronic Illness (CFQ-Cl; Gillanders et al., 2014; versão portuguesa de Trindade, Ferreira, \& Pinto-Gouveia, 2018). O CFQ-Cl avalia o nível de fusão cognitiva relacionada com o facto de se ter um diagnóstico de doença crónica e respetiva sintomatologia. Este questionário é composto por sete itens, que são respondidos através de uma escala de sete pontos, indo de 1 “Nunca verdadeiro" a 7 "Sempre verdadeiro". São exemplos de itens "Fico tão enredado/a ("preso/a") nos meus pensamentos em relação à minha doença que me torno incapaz de fazer as coisas que eu mais quero fazer" e "Sinto muita dificuldade em "largar" os meus pensamentos perturbadores, relacionados com a minha doença, mesmo quando sei que seria mais vantajoso fazêlo". Na versão portuguesa, o valor reportado para a sua consistência interna foi de 0,90 (Trindade, Ferreira, \& Pinto-Gouveia, 2018). No presente estudo foi encontrado um valor de alfa de Cronbach de 0,95.

Acceptance and Action Questionnaire-II (AAQ-II; Bond et al, 2011; versão portuguesa de Pinto-Gouveia et al., 2012). O AAQ-II destina-se a avaliar o evitamento experiencial (Bond et al, 2011). Este questionário integra sete itens, respondidos numa escala de sete pontos, em que 1 corresponde a "Nunca verdadeiro" e a 7 "Sempre verdadeiro". São exemplos de itens "As minhas experiências e memórias dolorosas dificultam que eu viva uma 
vida que valorize" e "As minhas preocupações atravessam-se no caminho do meu sucesso". Pontuações mais elevadas são indicadoras de maiores níveis de evitamento experiencial. A consistência interna, medida através do alfa de Cronbach na versão portuguesa, foi de 0,90 (Pinto-Gouveia et al., 2012). Neste estudo o alfa de Cronbach encontrado foi 0,95 .

Self-Compassion Scale (SCS; Neff, 2003; versão portuguesa de Castilho \& Pinto-Gouveia, 2011). A SCS é composta por 26 itens divididos em seis subescalas: calor/compreensão (e.g., "Sou tolerante com os meus erros e inadequações"); autocriticismo (e.g., "Desaprovo-me e faço julgamentos acerca dos meus erros e inadequações"); humanidade comum (e.g., "Tento ver os meus erros e falhas como parte da condição humana"); isolamento (e.g., "Quando penso acerca das minhas inadequações e defeitos sinto-me mais separado e desligado do resto do mundo"); mindfulness (e.g., "Quando alguma coisa dolorosa acontece tento ter uma visão equilibrada da situação") e sobreidentificação (e.g., "Quando alguma coisa me aborrece ou entristece deixo-me levar pelos meus sentimentos"). Os itens são respondidos através de uma escala de cinco pontos, sendo que 1 corresponde a "Quase nunca” e 5 "Quase sempre”. Relativamente à consistência interna na versão portuguesa, foi encontrado um valor de 0,89 para o total, variando de 0,73 a 0,84 relativamente às subescalas (Castilho \& Pinto-Gouveia, 2011). No presente estudo foram utilizados os índices compósitos de autocompaixão (somatório das subescalas de calor/compreensão, humanidade comum e mindfulness) e de autojulgamento (somatório das subescalas de autocriticismo, isolamento e sobreidentificação). O uso dos dois índices compósitos independentes que captam os aspetos positivos e negativos da autocompaixão é recomendado por Brenner et al. (2017), em vez de cálculo de um score total, o que é igualmente sugerido pela análise fatorial confirmatória conduzida por Costa et al. (2016). No presente estudo foram obtidos valores de alfa de Cronbach de 0,95 e de 0,96, para os índices de autocompaixão e de autojulgamento, respetivamente.

Chronic Illness-related Shame Scale (CISS; Trindade et al., 2017). A CISS é uma medida de vergonha especificamente relacionada com a doença crónica. Esta escala integra sete itens (e.g., "Sinto vergonha de falar com os outros sobre a minha doença ou sintomas"; "Sinto-me inseguro/a devido à minha doença"), que são respondidos mediante uma escala de cinco pontos, variando de 0 "Nunca verdadeiro" a 4 "Sempre verdadeiro". Os valores de consistência interna da versão portuguesa obtidos em diferentes amostras variaram entre 0,91 e 0,93 (Trindade et al., 2017). No presente estudo a CISS revelou um valor de alfa de Cronbach de 0,95.

\section{Procedimentos metodológicos}

O recrutamento dos participantes foi realizado através da APC. Esta associação de pacientes foi informada acerca do âmbito, objetivos e procedimento de recolha de dados definidos para o estudo, tendo considerado estarem assegurados os aspetos éticos e colaborado na divulgação deste junto dos seus associados. A recolha de dados foi efetuada online, entre março e abril de 2019, sendo disponibilizado um link com acesso ao conjunto dos instrumentos de autorresposta. Através deste link os participantes tomaram conhecimento do âmbito e objetivos do estudo, da natureza voluntária da participação que Ihes era solicitada e da garantia do anonimato e confidencialidade dos dados. Todos os participantes facultaram o seu consentimento informado, sendo esta uma condição obrigatória para prosseguirem para o preenchimento do protocolo. 


\section{Procedimentos analíticos}

Na descrição da amostra recorreu-se a estatística descritiva, sendo calculadas as médias e desvios padrão das variáveis contínuas e frequências e percentagens das variáveis categoriais, sociodemográficas e clínicas. As medidas de tendência central foram analisadas para as diferentes variáveis em estudo, sendo comparadas com as médias obtidas noutras amostras de pessoas com doença crónica através do cálculo de testes $t$ para uma amostra. Na comparação dos valores médios entre os participantes do sexo masculino e feminino, relativamente às diferentes variáveis em estudo, procedeu-se ao cálculo do teste Wilcoxon-Mann-Whitney. A existência de associações entre as variáveis idade, anos de escolaridade e duração do diagnóstico com as demais variáveis foi explorada através do cálculo de correlações de Pearson. Com o objetivo de identificar a contribuição independente dos diferentes fatores preditores (variáveis relativas aos processos de regulação emocional e vergonha associada à doença crónica) na explicação dos sintomas emocionais negativos (variáveis dependentes) foram conduzidas análises de regressão linear múltipla hierárquica. Ainda que o critério do tamanho da amostra não se tenha verificado (seriam necessários 82 sujeitos), uma vez que se tratava de uma amostra clínica, a análise foi efetuada atendendo a que não se observou multicolinearidade (todas as variáveis independentes revelaram valores de tolerância superiores a 0,10 e de VIF inferiores a 10; Tabachnick \& Fidell, 2013). De acrescentar que não se encontraram violações severas ao pressuposto da normalidade, sendo que os valores de assimetria foram $|<3|$ e de achatamento $|<10|$ (Kline, 2005). Relativamente às correlações, foram consideradas baixas ( $r$ entre 0,10 e 0,29), moderadas ( $r$ entre 0,30 e 0,49) e elevadas ( $r$ entre 0,50 e 1 ), de acordo com Pallant (2013). Em todas as análises foi considerado o nível de significância inferior a 0,05.

\section{Resultados}

Os valores médios das variáveis em estudo, mais precisamente dos sintomas emocionais negativos, dos processos relacionados com a regulação emocional, e da vergonha associada à doença crónica, assim como os referidos valores separadamente para homens e mulheres, são apresentados na Tabela 1.

Ao comparar os valores médios obtidos na presente amostra de pacientes com DC com os reportados por Trindade et al. (2019) numa amostra de pacientes com doença inflamatória do intestino (DII), também recrutada online, não se verificaram diferenças estatisticamente significativas nos sintomas de depressão ( $t=$ $1,65 ; p=0,104)$ e de stress $(t=-1,84 ; p=0,070)$, evidenciando os pacientes com DC mais sintomas de ansiedade $(t=2,53 ; p=0,014)$. Quanto à fusão cognitiva, a comparação dos resultados da presente amostra com os obtidos com pacientes com DII (Trindade, Ferreira, \& Pinto-Gouveia, 2018) não indicou a existência de diferenças estatisticamente significativas $(t=-1,23 ; p=0,224)$, o mesmo acontecendo na comparação dos níveis de vergonha associada à doença crónica $(t=-1,10 ; p=0,277)$ e ao evitamento experiencial $(t=-1,97 ; p=0,053)$ (Trindade et al., 2017). Por sua vez, a comparação da média do autojulgamento em pacientes com DC com as médias obtidas por Pinto-Gouveia et al. (2013) não revelou existirem diferenças relativamente aos encontrados numa amostra de pacientes com diversas doenças crónicas e numa amostra de pacientes com cancro, evidenciando estas duas amostras o mesmo valor de autojulgamento $(t=-1,88 ; p=0,064)$. 


\section{Tabela 1}

Análise Descritiva das Variáveis em Estudo na Amostra Total, nas Mulheres e nos Homens com Doença Celíaca

\begin{tabular}{lcccccc}
\hline & \multicolumn{2}{c}{$\begin{array}{c}\text { Amostra total } \\
(N=67)\end{array}$} & \multicolumn{2}{c}{$\begin{array}{c}\text { Mulheres } \\
(n=60)\end{array}$} & \multicolumn{2}{c}{$\begin{array}{c}\text { Homens } \\
(n=7)\end{array}$} \\
\hline & $M$ & $D P$ & $M$ & $D P$ & $M$ & $D P$ \\
\hline EADS-21 (Depressão) & 5,96 & 5,43 & 5,83 & 5,44 & 7,00 & 5,69 \\
EADS-21 (Ansiedade) & 5,39 & 4,14 & 5,20 & 4,04 & 7,00 & 5,10 \\
EADS-21 (Stress) & 8,30 & 5,15 & 8,18 & 5,21 & 9,29 & 4,89 \\
CFQ-CI & 16,28 & 9,52 & 16,37 & 9,30 & 15,57 & 12,05 \\
AAQ-II & 19,40 & 11,30 & 19,65 & 11,62 & 17,29 & 8,42 \\
SCS-COMP & 38,99 & 11,66 & 38,60 & 11,93 & 42,29 & 9,10 \\
SCS-JULG & 34,16 & 13,41 & 34,33 & 13,38 & 32,71 & 14,66 \\
CISS & 7,27 & 8,23 & 7,38 & 8,15 & 6,29 & 9,45 \\
\hline
\end{tabular}

Nota. EADS-21 = Escalas de Ansiedade, Depressão e Stress; CFQ-CI = Cognitive Fusion Questionnaire-Chronic IIIness; AAQ-II = Acceptance and Action Questionnaire- II; SCS-Comp = Self-Compassion Scale - Self-Compassion; SCS-Julg = Self-Compassion Scale - Self-Judgment; CISS = Chronic IIIness-related Shame Scale.

Tendo sido examinada a eventual existência de diferenças entre homens e mulheres relativamente a estas variáveis, não se observou a existência de diferenças estatisticamente significativas em nenhuma delas ( $p>$ $0,05)$.

Enquanto análise preliminar à condução de regressões lineares múltiplas hierárquicas, foi efetuado o cálculo de correlações entre as variáveis em estudo, sendo estes resultados reportados na Tabela 2.

\section{Tabela 2}

Correlações entre Sintomas Psicopatológicos, Processos de Regulação Emocional e Vergonha Relacionada com Doença Crónica Numa Amostra de Pessoas com Doença Celíaca

\begin{tabular}{lcccccccc}
\hline & 1 & 2 & 3 & 4 & 5 & 6 & 7 & 8 \\
\hline 1. EADS-21 (Depressão) & - & - & - & - & - & - & - & - \\
2. EADS-21 (Ansiedade) & $0,65^{* *}$ & - & - & - & - & - & - & - \\
3. EADS-21 (Stress) & $0,83^{* *}$ & $0,73^{* *}$ & - & - & - & - & - & - \\
4. CFQ-Cl & $0,55^{* *}$ & $0,42^{* *}$ & $0,50^{* *}$ & - & - & - & - & - \\
5. AAQ-II & $0,68^{* *}$ & $0,44^{* *}$ & $0,67 * *$ & $0,60 * *$ & - & - & - & - \\
6. SCS-Comp & $-0,34^{* *}$ & $-0,20$ & $-0,40^{* *}$ & $-0,13$ & $-0,38^{* *}$ & - & - & - \\
7. SCS-Julg & $0,75^{* *}$ & $0,52^{* *}$ & $0,72^{* *}$ & $0,55^{* *}$ & $0,84^{* *}$ & $-0,36^{* *}$ & - & - \\
8. CISS & $0,56^{* *}$ & $0,39^{* *}$ & $0,53^{* *}$ & $0,85^{* *}$ & $0,65^{* *}$ & $-0,21^{* *}$ & $0,59^{* *}$ & - \\
\hline
\end{tabular}

Nota. EADS-21 = Escalas de Ansiedade, Depressão e Stress; CFQ-Cl = Cognitive Fusion Questionnaire-Chronic IIIness; AAQ-II = Acceptance and Action Questionnaire- II; SCS-Comp = Self-Compassion Scale - Self-Compassion; SCS-Julg = Self-Compassion Scale - Self-Judgment; CISS = Chronic IIIness-related Shame Scale.

${ }^{*} p<0,05 ;{ }^{* *} p<0,01$.

Verificaram-se correlações estatisticamente significativas entre todas as variáveis em estudo, à exceção da associação entre o índice compósito de autocompaixão e os sintomas de ansiedade, e entre esse mesmo índice 
compósito e a fusão cognitiva relacionada com a doença crónica. Para além disso, foi analisada a existência de associações entre as variáveis sociodemográficas (idade e anos de escolaridade), a duração do diagnóstico e demais variáveis. Os resultados mostraram apenas a existência de uma correlação negativa estatisticamente significativa entre o tempo de diagnóstico e a vergonha relacionada com a doença crónica $(r=-0,29 ; p<0,050)$. Com o intuito de explorar quais os preditores dos sintomas depressivos em pessoas com doença celíaca, procedeu-se ao cálculo da regressão linear múltipla. Os processos relacionados com a regulação emocional (fusão cognitiva relacionada com a doença crónica, evitamento experiencial, índices compósitos de autocompaixão e de autojulgamento) foram integrados no primeiro bloco e a vergonha relacionada com a doença crónica inserida no segundo bloco, atendendo a que esta não corresponde a uma variável processo, mas sim a uma medida de sentimentos de vergonha associados especificamente à condição de doença crónica. O primeiro bloco explicou $57 \%$ da variância dos sintomas depressivos e o segundo bloco não contribuiu para o aumento da variância, não se revelando significativo (Tabela 3).

\section{Tabela 3}

Análise de Regressão Linear Múltipla dos Preditores (Processos de Regulação Emocional e Vergonha) dos Sintomas Depressivos Numa Amostra de Pessoas com Doença Celíaca

\begin{tabular}{|c|c|c|c|c|c|c|c|}
\hline Preditores & $r$ & $R_{2}$ Ajustado & $F$ & $p$ & $B$ & $t$ & $p$ \\
\hline Modelo 1 & 0,77 & 0,57 & 22,85 & $<0,001$ & & & \\
\hline CFQ-Cl & & & & & 0,19 & 1,81 & 0,075 \\
\hline AAQ-II & & & & & 0,07 & 0,45 & 0,653 \\
\hline SCS-Comp & & & & & $-0,08$ & $-0,10$ & 0,322 \\
\hline SCS-Julg & & & & & 0,56 & 3,72 & $<0,001$ \\
\hline Modelo 2 & 0,77 & 0,56 & 0,03 & 0,875 & & & \\
\hline CFQ-Cl & & & & & 0,17 & 1,07 & 0,287 \\
\hline AAQ-II & & & & & 0,07 & 0,42 & 0,678 \\
\hline SCS-Comp & & & & & $-0,09$ & $-0,98$ & 0,331 \\
\hline SCS-Julg & & & & & 0,55 & 3,67 & 0,001 \\
\hline CISS & & & & & 0,03 & 0,16 & 0,875 \\
\hline
\end{tabular}

Nota. CFQ-Cl = Cognitive Fusion Questionnaire-Chronic IIIness; AAQ-II = Acceptance and Action Questionnaire- II; SCS-Comp = Self-Compassion Scale Self-Compassion; SCS-Julg = Self-Compassion Scale - Self-Judgment; CISS = Chronic IIIness-related Shame Scale. Os valores a negrito indicam a existência de significância estatística.

De referir que apenas o índice compósito de autojulgamento se mostrou significativo na predição da depressão $(\beta=0,55 ; p=0,001)$.

Uma análise idêntica foi também conduzida, considerando num primeiro bloco a fusão cognitiva relacionada com a doença crónica, o evitamento experiencial, e o índice compósito de autojulgamento e num segundo bloco a vergonha relacionada com a doença crónica, na predição dos sintomas ansiosos. Como se pode observar na Tabela 4, o primeiro bloco explicou $27 \%$ da variância dos sintomas de ansiedade e o segundo bloco não contribui para o aumento da variância deste tipo de sintomas, não se mostrando significativo. 


\section{Tabela 4}

Análise de Regressão Linear Múltipla dos Preditores (Processos de Regulação Emocional e Vergonha) dos Sintomas Ansiosos Numa Amostra de Pessoas com Doença Celíaca

\begin{tabular}{lccccccc}
\hline Preditores & $r$ & R2Ajustado & $F$ & $p$ & $B$ & $t$ & $p$ \\
\hline Modelo 1 & 0,55 & 0,27 & 8,94 & $<0,001$ & & & \\
CFQ-Cl & & & & 0,22 & 1,62 & 0,110 \\
AAQ-II & & & & $-0,10$ & $-0,48$ & 0,631 \\
SCS-Julg & & & & $\mathbf{0 , 4 8}$ & $\mathbf{2 , 4 8}$ & $\mathbf{0 , 0 1 6}$ \\
\hline Modelo 2 & 0,55 & 0,26 & 0,13 & 0,718 & & & 0,186 \\
CFQ-Cl & & & & 0,27 & $\mathbf{1 , 3 4}$ & 0,683 \\
AAQ-II & & & & $-0,09$ & $-0,41$ & $\mathbf{0 , 0 1 6}$ \\
SCS-Julg & & & & $\mathbf{0 , 4 9}$ & $\mathbf{2 , 4 9}$ & $\mathbf{0 , 0 1 6}$ \\
CISS & & & & $-0,08$ & $-0,36$ & 0,718 \\
\hline
\end{tabular}

Nota. $\mathrm{CFQ}-\mathrm{Cl}=$ Cognitive Fusion Questionnaire-Chronic IIIness; AAQ-II =Acceptance and Action Questionnaire-II; SCS-Julg = Self-Compassion Scale Self-Judgment; CISS = Chronic IIIness-related Shame Scale. Os valores a negrito indicam a existência de significância estatística.

De modo semelhante ao sucedido com os sintomas de depressão, também no que se refere aos sintomas de ansiedade, o único preditor que se revelou significativo foi o autojulgamento $(\beta=0,49 ; p=0,016)$.

Relativamente aos sintomas de stress nas pessoas com doença celíaca, também num primeiro bloco da análise de regressão linear foram inseridos os processos relacionados com a regulação emocional, sendo a vergonha relacionada com a doença crónica adicionada num segundo bloco. O primeiro bloco explicou $54 \%$ da variância dos sintomas de stress e o segundo bloco não contribuiu para o aumento da variância deste tipo de sintomas, não revelando significância estatística (Tabela 5).

\section{Tabela 5}

Análise de Regressão Linear Múltipla dos Preditores (Processos de Regulação Emocional e Vergonha) dos Sintomas de Stress Numa Amostra de Pessoas com Doença Celíaca

\begin{tabular}{|c|c|c|c|c|c|c|c|}
\hline Preditores & $r$ & R2Ajustado & $F$ & $p$ & $B$ & $t$ & $p$ \\
\hline Modelo 1 & 0,75 & 0,54 & 20,24 & $<0,001$ & & & \\
\hline CFQ-Cl & & & & & 0,14 & 1,29 & 0,201 \\
\hline AAQ-II & & & & & 0,12 & 0,72 & 0,473 \\
\hline SCS-Comp & & & & & $-0,16$ & $-1,75$ & 0,085 \\
\hline SCS-Julg & & & & & 0,49 & 3,17 & 0,002 \\
\hline Modelo 2 & 0,75 & 0,53 & 0,06 & 0,812 & & & \\
\hline CFQ-Cl & & & & & 0,11 & 0,67 & 0,504 \\
\hline AAQ-II & & & & & 0,11 & 0,67 & 0,506 \\
\hline sCS-Comp & & & & & $-0,16$ & $-1,72$ & 0,091 \\
\hline SCS-Julg & & & & & 0,49 & 3,13 & 0,003 \\
\hline CISS & & & & & 0,04 & 0,24 & 0,812 \\
\hline
\end{tabular}

Nota. CFQ-Cl = Cognitive Fusion Questionnaire-Chronic IIIness; AAQ-II = Acceptance and Action Questionnaire- II; SCS-Comp = Self-Compassion Scale Self-Compassion; SCS-Julg = Self-Compassion Scale - Self-Judgment; CISS = Chronic IIIness-related Shame Scale. Os valores a negrito indicam a existência de significância estatística. 
Relativamente aos sintomas de stress, o único preditor significativo foi o autojulgamento (índice compósito de autocriticismo, isolamento e sobreidentificação; $\beta=0,49 ; p=0,003$ ).

\section{Discussão}

Este estudo pretendeu examinar o papel preditor de processos relacionados com a regulação emocional (fusão cognitiva, evitamento experiencial, autocompaixão e autojulgamento) e da vergonha relacionada com a doença crónica em indivíduos com doença celíaca.

O cálculo dos valores médios para as variáveis em estudo e a comparação destes com os encontrados noutras amostras de pacientes com doença crónica (e.g., DII) revelou que os pacientes com DC da presente amostra não apresentam diferenças em termos de sintomas de depressão e de stress, bem como dos níveis de evitamento experiencial, mas revelam mais sintomas de ansiedade (Trindade et al., 2019). A inexistência de diferenças estatisticamente significativas também se observou relativamente à fusão cognitiva e aos níveis de vergonha associada à doença crónica reportados em doentes com DII (Trindade, Marta-Simões et al., 2018). No caso do autojulgamento também não se verificaram diferenças entre os pacientes com DC e pacientes com diversas doenças crónicas ou pacientes com cancro (Pinto-Gouveia et al., 2013). Na sua globalidade, os resultados sugerem que os pacientes com DC não diferem de pacientes com outras patologias crónicas, no entanto, estes resultados poderão encontrar uma maior sustentação em estudos futuros com amostras maiores de pacientes com DC, assim como a exploração de resultados relativamente aos sintomas de ansiedade. Para além disso, hipotetizamos que o tamanho da amostra, a duração do diagnóstico, bem como as manifestações da própria doença poderão contribuir para os resultados encontrados no presente estudo. Efetivamente, é de salientar que as manifestações de doença não se traduzem em alterações da imagem corporal, avaliação negativa por parte dos outros, limitações major de natureza social, podendo ter um menor impacto nos sentimentos de vergonha.

Ao considerar a relação entre as variáveis sociodemográficas da idade e anos de escolaridade, os processos relacionados com a regulação emocional e a vergonha relativa à doença crónica, não foram encontradas associações significativas. Assim, o recurso a diferentes processos para regular as emoções em pacientes com DC parece não depender da idade ou do nível de escolaridade dos pacientes. Estudos anteriores têm apontado resultados diversos relativamente a estes aspetos. Por exemplo, Carvalho et al. (2019) referem que, num estudo de natureza longitudinal realizado numa amostra de pacientes com dor crónica, as variáveis demográficas, como a idade ou os anos de escolaridade não se revelaram associadas com os sintomas de depressão em nenhum dos momentos de avaliação considerados no estudo. No entanto, Pinto-Gouveia et al. (2013), reportaram a existência de uma correlação negativa entre o nível de escolaridade e o autojulgamento, os sintomas de depressão, ansiedade e stress, num grupo de pacientes com diversas doenças crónicas e num grupo de pacientes com cancro. Assim, ainda que a associação entre os anos de escolaridade, a regulação emocional e os sintomas psicopatológicos possa vir a ser alvo de análise em estudos futuros, é de referir que na nossa amostra a média de anos de escolaridade dos participantes se situou ao nível do ensino superior, ao passo que a indicada no estudo acima referido se localizava no 3 o ciclo do ensino básico.

Tendo em conta a relação entre a duração do diagnóstico e as variáveis analisadas, apenas foi encontrada uma correlação negativa baixa com a vergonha associada à doença crónica. Esta poderá ser compreendida se 
atendemos a que um maior tempo de vivência com a DC poderá conduzir a uma diminuição dos sentimentos de vergonha resultantes da experiência da doença, face a uma maior adaptação e normalização das manifestações da doença e das implicações da DIG.

No presente estudo, a análise de eventuais diferenças entre homens e mulheres no tocante às diferentes variáveis consideradas não revelou a existência de diferenças estatisticamente significativas em nenhuma das variáveis. Com efeito, a existência de maiores níveis de sintomas psicopatológicos em mulheres comparativamente com homens encontra-se largamente documentada (American Psychiatric Association, 2014). Já no que se refere aos processos relacionados com a regulação emocional, os resultados de estudos anteriores não são conclusivos. Por exemplo, Dinis et al. (2015) não encontraram diferenças no evitamento experiencial, mas referem que as mulheres apresentaram valores mais elevados de fusão cognitiva comparativamente com os homens. Já Ruiz et al. (2017), num estudo que englobou diversas amostras, reportam que em algumas existem diferenças nas pontuações médias de um instrumento de fusão cognitiva, enquanto que noutras tal não se verifica. Por sua vez Galhardo et al. (2013) relatam a existência de maiores níveis de autojulgamento e de vergonha nas mulheres com um diagnóstico de infertilidade, mas não indicam a existência de diferenças entre os sexos relativamente à autocompaixão. Independentemente da literatura não ser conclusiva a este respeito, há que referir que a presente amostra de pacientes com DC é reduzida e que os homens são em número ainda mais reduzido. Como tal, consideramos que numa amostra de maior dimensão global, em que ambos os sexos se encontrem representados com maior equivalência, este resultado poderá ser diferente.

Relativamente ao principal objetivo deste estudo, o índice compósito de autojulgamento, que integra as componentes de autocriticismo, isolamento e sobreidentificação, revelou-se o único preditor significativo dos sintomas emocionais negativos de depressão, ansiedade e stress. Estes resultados sugerem que quando os pacientes evidenciam maior autocritica, se isolam e se sobreidentificam com a sua doença, têm mais probabilidade de desenvolver sintomas de depressão, ansiedade e stress. Efetivamente, um padrão idêntico já tinha sido identificado num estudo anterior que comparou sujeitos com diversas doenças crónicas (hipertensão, insuficiência cardíaca, artrite reumatoide, psoríase, osteoporose, doenças alérgicas, hipertiroidismo, doença de Crohn, bronquite asmática e glaucoma), controlos saudáveis e pacientes com cancro, no qual o autojulgamento foi também reportado como o único preditor significativo dos sintomas de depressão e de stress no grupo de pacientes com um diagnóstico de doença crónica (Pinto-Gouveia et al., 2013). De modo idêntico, Kane et al. (2018) observaram, em pacientes com diabetes tipo 2, que as dimensões negativas da autocompaixão, que correspondem ao índice compósito de autojulgamento, se associavam com o stress inerente à diabetes. Assim, também na DC, a adoção de uma atitude mais ajuizadora, mais autocrítica, a visão de si como isolado dos outros, e uma sobreidentificação com os problemas que a doença pode acarretar, parecer ser facilitadores do surgimento e manutenção de sintomas psicopatológicos.

Na globalidade, os resultados sugerem que em pacientes com DC, as abordagens terapêuticas que fomentam a redução do autojulgamento, como é o caso da Terapia Focada na Compaixão (TFC), podem ser particularmente adequadas no que respeita à diminuição dos sintomas emocionais negativos. Esta abordagem terapêutica transdiagnóstica integra contributos da psicologia evolucionária, social, desenvolvimental e Budista, assim como das neurociências. A TFC tem o intuito de desenvolver motivações e competências compassivas que, por sua vez, contribuem para uma maior abertura e tolerância face a dificuldades (como as que tendem a resultar da experiência de doença crónica) (Gilbert, 2014). De mencionar que a TFC tem demonstrado efeitos benéficos na 
depressão, ansiedade e stress (Leaviss \& Uttley, 2014), promovendo o bem-estar e a diminuição dos níveis de stress em pessoas com doença crónica (Sirois \& Rowse, 2016). De acrescentar que, para além da TFC se mostrar eficaz na redução de sintomas psicopatológicos, há a referir que um dos mecanismos associados a esta redução é precisamente o da diminuição dos níveis de autocriticismo (Cuppage et al., 2018). De notar ainda que a TFC tem vindo a ser descrita como fácil de compreender, bem tolerada, útil e conducente a uma melhoria das dificuldades psicológicas em contextos naturalistas (Judge et al., 2012). Tal é válido, mesmo num formato de autoajuda guiada (Sommers-Spijkerman et al., 2018), o que facilita a sua aplicação em diversas populações, sendo apontada como uma estratégia de promoção de saúde mental promissora.

Na análise dos resultados deste estudo há que atender a algumas limitações. Em primeiro lugar, o estudo foi conduzido numa amostra de tamanho reduzido, não assegurando a representatividade dos pacientes com DC. Assim, investigações futuras deverão ser realizadas em amostras de maior dimensão e, como já referido, com um maior equilíbrio entre participantes do sexo masculino e feminino. Outra limitação prende-se com o procedimento de recolha dos dados. Por um lado, os participantes foram recrutados através de uma associação de pacientes, sendo que a sua participação numa entidade deste tipo pode traduzir uma melhor adaptação à sua condição de doença e contribuir para níveis mais baixos de psicopatologia. Por outro lado, a recolha de dados num formato online fez com que o diagnóstico de DC fosse autorreportado, não tendo havido consulta de processos médicos.

\section{Conclusão}

Não obstante as limitações indicadas, o estudo contribuiu para uma melhor compreensão do papel dos processos relacionados com a regulação emocional em pessoas com DC e sua ligação com sintomas emocionais negativos. Neste contexto, foi identificado o autojulgamento como um alvo de intervenção relevante nestes pacientes, sendo que a recomendação da existência de apoio psicológico destinado a sujeitos com DC havia já sido apontada (e.g., Addolorato, 2001; Addolorato et al., 2004; Zarkadas et al., 2006). Mais especificamente, a integração de aspetos relacionados com as competências de regulação emocional, nas intervenções terapêuticas, contribui para a eficácia das referidas intervenções (Berking et al., 2008; Wierenga et al., 2017), o que parece poder também aplicar-se em pacientes com DC.

Agradecimentos | Acknowledgements: Os autores gostariam de agradecer à Associação Portuguesa de Celíacos (APC) pela colaboração na divulgação do estudo para efeitos de recolha da amostra clínica.

Conflito de interesses | Conflict of interest: nenhum | none.

Fontes de financiamento | Funding sources: nenhuma | none.

Contributos: MF: Pesquisa bibliográfica; Revisão da literatura; Preparação das medidas e escrita do protocolo; Recrutamento dos participantes; Análise estatística; Escrita do manuscrito. AG: Desenho do estudo; Preparação das medidas e escrita do protocolo; Análise estatística; Supervisão da escrita do manuscrito e aprovação do manuscrito final. IMC: Análise estatística; Revisão final e aprovação do manuscrito. 


\section{Referências}

Addolorato, G. (2001). Anxiety but not depression decreases in coeliac patients after one-year gluten-free diet: $A$ longitudinal study. Scandinavian Journal of Gastroenterology, 36(5), 502-506. https://doi.org/10.1080/00365520119754

Addolorato, G., De Lorenzi, G., Abenavoli, L., Leggio, L., Capristo, E., \& Gasbarrini, G. (2004). Psychological support counselling improves gluten-free diet compliance in coeliac patients with affective disorders. Alimentary Pharmacology and Therapeutics, 20(7), 777-782. https://doi.org/10.1111/j.1365-2036.2004.02193.x

American Psychiatric Association. (2014). Manual de diagnóstico e estatística das perturbações mentais (5a ed.). Climepsi Editores.

Associação Portuguesa de Celíacos. (2019). Prevalência e incidência [Prevalence and incidence]. https://www.celiacos.org.pt/o-que-e/

Bai, D., Brar, P., Holleran, S., Ramakrishnan, R., \& Green, P. H. R. (2005). Effect of gender on the manifestations of celiac disease: Evidence for greater malabsorption in men. Scandinavian Journal of Gastroenterology, 40(2), $183-187$. https://doi.org/10.1080/00365520510011498

Berking, M., Wupperman, P., Reichardt, A., Pejic, T., Dippel, A., \& Znoj, H. (2008). Emotion-regulation skills as a treatment target in psychotherapy. Behaviour Research and Therapy, 46(11), $1230-1237$. https://doi.org/10.1016/j.brat.2008.08.005

Bond, F. W., Hayes, S. C., Baer, R. A., Carpenter, K. M., Guenole, N., Orcutt, H. K., Waltz, T., \& Zettle, R. (2011). Preliminary psychometric properties of the Acceptance and Action Questionnaire-Il: A revised measure of psychological inflexibility and experiential avoidance. Behavior Therapy, 42(4), 676-688. https://doi.org/10.1016/j.beth.2011.03.007

Brenner, R. E., Heath, P. J., Vogel, D. L., \& Credé, M. (2017). Two is more valid than one: Examining the factor structure of the Self-Compassion Scale (SCS). Journal of Counseling Psychology, 64(6), 696-707. https://doi.org/10.1037/cou0000211

Butterworth, J., \& Los, L. (2019). Coeliac disease. Medicine, 47(5). 314-319. https://doi.org/10.1016/j.mpmed.2019.02.003

Carvalho, S. A., Trindade, I. A., Gillanders, D., Pinto-Gouveia, J., \& Castilho, P. (2019). Cognitive fusion and depressive symptoms in women with chronic pain: A longitudinal growth curve modelling study over 12-months. Clinical Psychology \& Psychotherapy, 26(5), 616-625. https://doi.org/10.1002/cpp.2386

Castilho, P., \& Gouveia, J. P. (2011). Auto-compaixão: Estudo da validação da versão portuguesa da Escala da AutoCompaixão e da sua relação com as experiências adversas na infância, a comparação social e a psicopatologia [Selfcompassion: Study of the validation of the Portuguese version of the Self-Compassion Scale and its relationship with adverse childhood experiences, social comparison, and psychopathology]. Psychologica, (54), $203-230$. https://impactum-journals.uc.pt/psychologica/article/view/1106

Catassi, C., \& Lionetti, E. (2020). Celiac disease. In E. J. Kuipers (Ed.), Encyclopedia of gastroenterology (2nd ed., pp. 447452). https://doi.org//10.1016/B978-0-12-801238-3.65624-6

Celiac Disease Foundation. (2019). What is celiac disease?. https://celiac.org/about-celiac-disease/what-is-celiacdisease/

Chawla, N., \& Ostafin, B. (2007). Experiential avoidance as a functional dimensional approach to psychopathology: An empirical review. Journal of Clinical Psychology, 63(9), 871-890. https://doi.org/10.1002/jclp.20400

Cossu, G., Carta, M. G., Contu, F., Mela, Q., Demelia, L., Elli, L., \& Dell'Osso, B. (2017). Coeliac disease and psychiatric comorbidity: Epidemiology, pathophysiological mechanisms, quality-of-life, and gluten-free diet effects. International Review of Psychiatry, 29(5), 489-503. https://doi.org/10.1080/09540261.2017.1314952

Costa, J., Marôco, J., Pinto-Gouveia, J., Ferreira, C., \& Castilho, P. (2016). Validation of the psychometric properties of the Self-Compassion Scale. Testing the factorial validity and factorial invariance of the measure among borderline personality disorder, anxiety disorder, eating disorder and general populations. Clinical Psychology \& Psychotherapy, 23(5), 460-468. https://doi.org/10.1002/cpp.1974 
Cuppage, J., Baird, K., Gibson, J., Booth, R., \& Hevey, D. (2018). Compassion focused therapy: Exploring the effectiveness with a transdiagnostic group and potential processes of change. British Journal of Clinical Psychology, 57(2), 240254. https://doi.org/10.1111/bjc.12162

Dahl, J., Lundgren, T., Plumb, J., \& Stewart, I. (2009). The art and science of valuing in psychotherapy: Helping clients discover, explore, and commit to valued action using Acceptance and Commitment Therapy. New Harbinger Publications.

Dinis, A., Carvalho, S., Gouveia, J. P., \& Estanqueiro, C. (2015). Shame memories and depression symptoms: The role of cognitive fusion and experiential avoidance. International Journal of Psychology and Psychological Therapy, 15(1), 63-86. https://www.redalyc.org/articulo.oa?id=56038720004

Dowd, A. J., Jung, M. E., Chen, M. Y., \& Beauchamp, M. R. (2015). Prediction of adherence to a gluten-free diet using protection motivation theory among adults with coeliac disease. Journal of Human Nutrition and Dietetics, 29(3), 391-398. https://doi.org/10.1111/jhn.12321

Galhardo, A., Cunha, M., Pinto-Gouveia, J., \& Matos, M. (2013). The mediator role of emotion regulation processes on infertility-related stress. Journal of Clinical Psychology in Medical Settings, 20(4), $497-507$. https://doi.org/10.1007/s10880-013-9370-3

Gilbert, P. (2002). Body shame: A biopsychosocial conceptualization and overview with treatment implications. In P. Gilbert \& J. Miles (Eds.), Body shame: Conceptualization, research and treatment (pp. 3-47). Routledge.

Gilbert, P. (2007). The evolution of shame as a marker for relationship security. In J. L. Tracy, R. W. Robins, \& J. P. Tangney (Eds.), The self-conscious emotions: Theory and research (pp. 283-309). Guilford Press.

Gilbert, P. (2014). The origins and nature of compassion focused therapy. British Journal of Clinical Psychology, 53(1), 6-41. https://doi.org/10.1111/bjc.12043

Gillanders, D. T., Bolderston, H., Bond, F. W., Dempster, M., Flaxman, P. E., Campbell, L., Kerr, S., Tansey, L., Noel, P., Ferenbach, C., Masley, S., Roach, L., Lloyd, J., May, L., Clarke, S., \& Remington, B. (2014). The development and initial validation of the Cognitive Fusion Questionnaire. Behavior Therapy, 45(1), 83-101. https://doi.org/10.1016/j.beth.2013.09.001

Hall, N. J., Rubin, G., \& Charnock, A. (2009). Systematic review: Adherence to a gluten-free diet in adult patients with coeliac disease. Alimentary Pharmacology \& Therapeutics, 30(4), 315-330. https://doi.org/10.1111/j.13652036.2009.04053.x

Hayes, S. C., Wilson, K. G., Gifford, E. V., Follette, V. M., \& Strosahl, K. (1996). Experiential avoidance and behavioral disorders: A functional dimensional approach to diagnosis and treatment. Journal of Consulting and Clinical Psychology, 64(6), 1152-1168. https://doi.org/10.1037/0022-006x.64.6.1152

Hayes, S. C., Luoma, J. B., Bond, F. W., Masuda, A., \& Lillis, J. (2006). Acceptance and commitment therapy: Model, processes and outcomes. Behaviour Research and Therapy, 44(1), 1-25. https://doi.org/10.1016/j.brat.2005.06.006

Judge, L., Cleghorn, A., McEwan, K., \& Gilbert, P. (2012). An exploration of group-based compassion focused therapy for a heterogeneous range of clients presenting to a community mental health team. International Journal of Cognitive Therapy, 5(4), 420-429. https://doi.org/10.1521/ijct.2012.5.4.420

Kane, N. S., Hoogendoorn, C. J., Tanenbaum, M. L., \& Gonzalez, J. S. (2018). Physical symptom complaints, cognitive emotion regulation strategies, self-compassion and diabetes distress among adults with Type 2 diabetes. Diabetic Medicine, 35(12), 1671-1677. https://doi.org/10.1111/dme.13830

Kline, R. B. (2005). Principles and practice of structural equation modeling (2nd ed.). Guilford Press.

Leaviss, J., \& Uttley, L. (2014). Psychotherapeutic benefits of compassion-focused therapy: An early systematic review. Psychological Medicine, 45(5), 927-945. https://doi.org/10.1017/s0033291714002141

Leonard, M. M., Sapone, A., Catassi, C., \& Fasano, A. (2017). Celiac disease and nonceliac gluten sensitivity. JAMA, 318(7), 647-656. https://doi.org/10.1001/jama.2017.9730

Levin, M. E., MacLane, C., Daflos, S., Seeley, J. R., Hayes, S. C., Biglan, A., \& Pistorello, J. (2014). Examining psychological inflexibility as a transdiagnostic process across psychological disorders. Journal of Contextual Behavioral Science, 3(3), 155-163. https://doi.org/10.1016\%2Fj.jcbs.2014.06.003 
Lovibond, P., \& Lovibond, S. (1995). The structure of negative emotional states: Comparison of the depression anxiety stress scales (DASS) with the Beck Depression and Anxiety Inventories. Behaviour Research and Therapy, 33(3), 335343. https://doi.org/10.1016/0005-7967(94)00075-U

Ludvigsson, J. F., Card, T., Ciclitira, P. J., Swift, G. L., Nasr, I., Sanders, D. S., \& Ciacci, C. (2015). Support for patients with celiac disease: A literature review. United European Gastroenterology Journal, 3(2), $146-159$. https://doi.org/10.1177/2050640614562599

Mariné, M., Farre, C., Alsina, M., Vilar, P., Cortijo, M., Salas, A., Fernández-Banãres, F., Rosinach, M., Santaolalla, R., Loras, C., Marquès, T., Cusí, V., Hernández, M. I., Carrasco, A., Ribes, J., Viver, J. M., \& Esteve, M. (2011). The prevalence of coeliac disease is significantly higher in children compared with adults. Alimentary Pharmacology \& Therapeutics, 33(4), 477-486. https://doi.org/10.1111/j.1365-2036.2010.04543.x

Masuda, A., Mandavia, A., \& Tully, E. C. (2014). The role of psychological inflexibility and mindfulness in somatization, depression, and anxiety among Asian Americans in the United States. Asian American Journal of Psychology, 5(3), 230-236. https://doi.org/10.1037/a0034437

Matos, M., \& Pinto-Gouveia, J. (2010). Shame as a traumatic memory. Clinical Psychology and Psychotherapy, 17(4), 299-312. https://doi.org/10.1002/cpp.659

Melicharova, J., Slavik, M., \& Cervinkova, M. (2016). Psychological aspects in celiac disease: Step by step from symptoms to daily life with celiac disease. International Journal of Celiac Disease,4(2), 64-67. http://pubs.sciepub.com/ijcd/4/2/3/index.html

Nachman, F., del Campo, M. P., González, A., Corzo, L., Vázquez, H., Sfoggia, C., Smecuol, E., Pinto-Sánchez, M. I. P., Niveloni, S., Sugai, E., Maurino, E., \& Bai, J. C. (2010). Long-term deterioration of quality of life in adult patients with celiac disease is associated with treatment noncompliance. Digestive and Liver Disease, 42(10), 685691. https://doi.org/10.1016/j.dld.2010.03.004

Neff, K. (2003). The development and validation of a scale to measure self-compassion. Self and Identity, 2(3), 223-250. https://doi.org/10.1080/15298860309027

Neff, K. D. (2019). Setting the record straight about the Self-Compassion Scale. Mindfulness, 10(1), $200-202$. https://doi.org/10.1007/s12671-018-1061-6

Pais-Ribeiro, J. L., Honrado, A., \& Leal, I. (2004). Contribuição para o estudo da adaptação portuguesa das Escalas de Ansiedade, Depressão e Stress (EADS) de 21 itens de Lovibond e Lovibond [Contribution to the adaptation study of the Portuguese adaptation of the Lovibond and Lovibond Depression, Anxiety and Stress Scales (EADS) with 21 items]. Psicologia, Saúde \& Doenças, 5(2), 229-239. https://bit.ly/3eDt5gg

Pallant, J. (2013). SPSS survival manual: A step by step guide to data analysis using IBM SPSS (5th ed.). Allen \& Unwin.

Pinto-Gouveia, J., Gregório, S., Dinis, A., \& Xavier, A. (2012). Experiential avoidance in clinical and non-clinical samples: AAQ-II Portuguese version. International Journal of Psychology \& Psychological Therapy, 12(2), $139-156$. https://bit.ly/3bpFKkJ

Pinto-Gouveia, J., Duarte, C., Matos, M., \& Fráguas, S. (2013). The protective role of self-compassion in relation to psychopathology symptoms and quality of life in chronic and in cancer patients. Clinical Psychology \& Psychotherapy, 21(4), 311-323. https://doi.org/10.1002/cpp.1838

Ruiz, F. J., Suárez-Falcón, J. C., Riano-Hernández, D., \& Gillanders, D. (2017). Psychometric properties of the Cognitive Fusion Questionnaire in Colombia. Revista Latinoamericana de Psicología, 49(1), 80-87. https://doi.org/10.1016/j.rlp.2016.09.006

Siniscalchi, M., lovino, P., Tortora, R., Forestiero, S., Somma, A., Capuano, L., Franzese, M. D., Sabbatini, F., \& Ciacci, C. (2005). Fatigue in adult coeliac disease. Alimentary Pharmacology and Therapeutics, 22(5), $489-494$. https://doi.org/10.1111/j.1365-2036.2005.02619.x

Sirois, F. M., Molnar, D. S., \& Hirsch, J. K. (2015). Self-compassion, stress, and coping in the context of chronic illness. Self and Identity, 14(3), 334-347. https://doi.org/10.1080/15298868.2014.996249

Sirois, F. M., \& Rowse, G. (2016). The role of self-compassion in chronic illness care. Journal of Clinical Outcomes Management, 23(11), 521-527. https://www.mdedge.com/jcomjournal/article/146122/role-self-compassionchronic-illness-care 
Smith, D. F., \& Gerdes, L. U. (2012). Meta-analysis on anxiety and depression in adult celiac disease. Acta Psychiatrica Scandinavica, 125(3), 189-193. https://doi.org/10.1111/j.1600-0447.2011.01795.x

Sommers-Spijkerman, M. P. J., Trompetter, H. R., Schreurs, K. M. G., \& Bohlmeijer, E. T. (2018). Compassion-focused therapy as guided self-help for enhancing public mental health: A randomized controlled trial. Journal of Consulting and Clinical Psychology, 86(2), 101-115. https://psycnet.apa.org/doi/10.1037/ccp0000268

Tabachnick, B. G., \& Fidell, L. S. (2013). Using multivariate statistics (6th ed.). Pearson Education Inc.

Trindade, I. A. (2018). Emotion regulation and chronic illness: The roles of acceptance, mindfulness and compassion in physical and mental health [Doctoral dissertation, Universidade de Coimbra]. Repositório científico Universidade de Coimbra. https://eg.uc.pt/handle/10316/87592

Trindade, I. A., Ferreira, C., \& Pinto-Gouveia, J. (2017). Chronic illness-related shame: Development of a new scale and novel approach for IBD patients' depressive symptomatology. Clinical Psychology \& Psychotherapy, 24(1), 255263. https://doi.org/10.1002/cpp.2035

Trindade, I. A., Ferreira, C., \& Pinto-Gouveia, J. (2018). Assessment of chronic illness-related cognitive fusion: Preliminary development and validation of a new scale with an IBD sample. Journal of Clinical Psychology in Medical Settings, 25(4), 356-366. https://doi.org/10.1007/s10880-017-9536-5

Trindade, I. A., Keefer, L., Portela, F., Ferreira, C., \& Pinto-Gouveia, J. (2019). Measuring structural model invariance across internet-recruited and hospital-recruited IBD patients: Experiential avoidance's effect on psychopathological symptoms. Current Psychology, 1-8. https://doi.org/10.1007/s12144-019-00281-2

Trindade, I. A., Marta-Simões, J., Ferreira, C., \& Pinto-Gouveia, J. (2018). Chronic illness-related cognitive fusion explains the impact of body dissatisfaction and shame on depression symptoms in breast cancer patients. Clinical Psychology \& Psychotherapy, 25(6), 886-893. https://doi.org/10.1002/cpp.2323

Wierenga, K. L., Lehto, R. H., \& Given, B. (2017). Emotion regulation in chronic disease populations: An integrative review. Research and Theory for Nursing Practice, 31(3), 247-271. https://doi.org/10.1891/1541-6577.31.3.247

Zarkadas, M., Cranney, A., Case, S., Molloy, M., Switzer, C., Graham, I. D., Butzner, J. D., Rashid, M., Warren, R. E., \& Burrows, V. (2006). The impact of a gluten-free diet on adults with coeliac disease: Results of a national survey. Journal of Human Nutrition and Dietetics, 19(1), 41-49. https://doi.org/10.1111/j.1365-277x.2006.00659.x

Zingone, F., Swift, G. L., Card, T. R., Sanders, D. S., Ludvigsson, J. F., \& Bai, J. C. (2015). Psychological morbidity of celiac disease: A review of the literature. United European Gastroenterology Journal, 3(2), $136-145$. https://doi.org/10.1177/2050640614560786 